IdeAs

Idées d'Amériques

$3 \mid 2012$

L'alimentation dans les Amériques au prisme des sciences sociales

\title{
Le mal du pays comme un goût à la bouche. Pratiques alimentaires chez les migrants de Patamban (Michoacán, Mexique) habitant aux États-Unis
}

Tasting Homesickness : Dietary habits of Patamban (Michoacán, Mexico)

Immigrants Residing in the United States

La nostalgia del país, como un sabor en la boca. Prácticas de alimentación de

migrantes de Patamban (Michoacán, México) que viven en Estados Unidos

Frida CALDERON-BONY

\section{OpenEdition}

Édition électronique

URL : https://journals.openedition.org/ideas/490

DOI : 10.4000/ideas.490

ISSN : 1950-5701

Éditeur

Institut des Amériques

Référence électronique

Frida CALDERON-BONY, "Le mal du pays comme un goût à la bouche. Pratiques alimentaires chez les migrants de Patamban (Michoacán, Mexique) habitant aux États-Unis », IdeAs [En ligne], 3 | 2012, mis en ligne le 13 décembre 2012, consulté le 20 octobre 2022. URL : http://journals.openedition.org/ ideas/490 ; DOI : https://doi.org/10.4000/ideas.490

Ce document a été généré automatiquement le 20 octobre 2022

Creative Commons - Attribution - Pas d'Utilisation Commerciale - Pas de Modification 4.0 International - CC BY-NC-ND 4.0

https://creativecommons.org/licenses/by-nc-nd/4.0/ 


\section{Le mal du pays comme un goût à la bouche. Pratiques alimentaires chez les migrants de Patamban (Michoacán, Mexique) habitant aux États-Unis}

Tasting Homesickness : Dietary habits of Patamban (Michoacán, Mexico)

Immigrants Residing in the United States

La nostalgia del país, como un sabor en la boca. Prácticas de alimentación de migrantes de Patamban (Michoacán, México) que viven en Estados Unidos

Frida CALDERON-BONY

« L'aliment construit le mangeur : il est donc naturel que le mangeur cherche à se construire

en mangeant » Claude Fischler (2001)

\section{Introduction}

Parmi les travaux qui traitent des liens entre l'alimentation et la migration, un grand nombre d'entre eux porte sur les fonctions socioculturelles et identitaires de l'alimentation. Il est en effet reconnu que les « immigrants conservent dans une large mesure leur style alimentaire et culinaire » dans les sociétés de destination (Fischler, 2001 : 152). De même, la mobilité des humains et les pratiques alimentaires se déplacent d'un pays à un autre, provoquant la mise en contact de registres culinaires différents au sein des habitudes alimentaires des migrants. En explorant les enjeux qui traversent les processus identitaires à partir de l'alimentation, ces travaux s'intéressent, par exemple, à la manière dont les modes d'approvisionnement des produits dits « du pays » 
affectent les changements alimentaires des sujets dans les sociétés d'immigration (Bouly de Lesdain, 2002) ; ou bien en quoi ils favorisent la mise en place et le maintien de réseaux sociaux à travers les dons et le commerce alimentaire entre les lieux d'origine et les lieux de destination (Lestage, 2008). Dans un autre registre, Jean-Pierre Hassoun (2010) analyse la consommation dans les restaurants tenus par des migrants à New York et, en centrant son questionnement sur la consommation des plats en dehors de leur contexte d'origine, se demande si les plats proposés dans ces restaurants sont consommés tels qu'ils le seraient dans les espaces d'où ils viennent. Cet auteur propose une distinction entre la circulation des aliments et le transfert alimentaire, comme un moyen qui permettrait de comprendre la façon dont «l'autre inventé pour soi ou en fonction du soi » structure la consommation en dehors du contexte d'origine.

Dans la continuité de ces perspectives de recherche, nous voudrions ici approfondir notre réflexion sur le lien entre l'alimentation et la construction identitaire des individus dans un contexte migratoire. Nous nous proposons d'interroger la manière dont le migrant négocie son altérité à travers son alimentation, aussi bien face aux membres de la société d'accueil, que face à ceux de sa société d'origine. Nous verrons, en analysant le cas des migrants du village de Patamban (Michoacán, Mexique) qui partent aux États-Unis, que c'est à partir de la proximité et/ou distance avec les différents registres culinaires (culture d'origine et culture de destination) que les individus définissent une "nouvelle manière de manger ». Cette dernière leur sert ensuite de support dans la construction de leur identité de migrant.

C'est d'abord l'évocation récurrente des plats mexicains, lors des entretiens que nous avons réalisés avec des migrants aux États-Unis, qui nous a poussée à nous interroger sur l'importance de s'alimenter « comme au pays ». Pourquoi les migrants manifestentils une telle nostalgie des aliments de leur pays si les plats qu'ils consomment aux ÉtatsUnis sont souvent mexicains? Si pour l'anthropologue en situation d'observation, les repas pris au domicile par les familles sont clairement des repas mexicains dans la mesure où la préparation et les ingrédients composant les plats sont les mêmes qu'au Mexique; pour les migrants, au contraire, la nourriture mexicaine fait partie des nostalgies les plus souvent évoquées dans leurs récits de vie quand ils sont à l'étranger. Le migrant vit un manque dans le sens où il considère que les plats " $\mathrm{d}^{\prime}$ ici » (lieu de destination) n'ont pas le même goût que « là-bas » (au village d'origine). C'est à partir de l'évocation de certains plats régionaux ou locaux que les migrants semblent nourrir leurs souvenirs et entretenir une mémoire commune, qui leur permet de fonder des liens dans la distance. «La nourriture devient alors un véritable discours du passé et le récit nostalgique du pays, de la région, de la ville ou du village où l'on est né » (de Certeau et. al., 1994: 259). C'est la manifestation constante d'une envie de vouloir manger « comme au pays » qui permet de recréer sans cesse l'attachement affectif avec la culture d'origine.

4 Dans le même temps se manifestent également de nouvelles manières de manger. Ainsi, le migrant exprime, par ces nouvelles manières de manger et ces nouveaux goûts culinaires, une nouvelle condition économique à laquelle il a pu accéder grâce à la migration. En parlant de ses nouveaux goûts culinaires, il cherche essentiellement à marquer une différence avec le passé connu dans son village d'origine, un passé marqué par la pauvreté et le souvenir d'avoir vécu avec moins de ressources et une alimentation moins variée. Dans ce cas, les aliments aident à évaluer le nouveau statut social des familles. «La nourriture est un repère, il permet au souvenir de retracer la 
hiérarchie sociale »(Bahloul, 1992: 112). C'est alors en intégrant de nouveaux aliments à son régime alimentaire que le migrant affiche un nouveau statut social. Il cherche à se distinguer, tout en montrant que c'est grâce à son expérience migratoire qu'il mange d'une nouvelle manière.

5 Au delà de l'identification d'une permanence ou non de la cuisine mexicaine dans le régime alimentaire des migrants aux États-Unis, la consommation des aliments identifiés comme appartenant soit au pays d'origine, soit au pays de destination, constitue un repère permettant au migrant de négocier sa position sociale par rapport à son pays d'origine, pays qu'il a quitté et où il ne vit plus ; mais aussi avec la société qu'il a rejoint, où il vit à présent et où il mange tous les jours. Le maintien d'une tradition culinaire d'origine mexicaine et la transformation des pratiques alimentaires quotidiennes, influencées par la nourriture étatsunienne, forment en réalité un univers gastronomique fait de ruptures et de continuités. Les notes de notre journal de terrain manifestent ce mélange :

15 avril 2005. « C'est mon premier jour à Fort Pierce (Floride, États-Unis). J'habite chez Maria. Ce soir-là, à l'heure du dîner, elle me propose un verre de lait et une fina $a^{1}$ de Patamban (son oncle est arrivé la veille du village et leur a apporté ce type de pain). Le jour suivant, le repas de midi pris à la maison est composé de : haricots noirs, tortillas de maïs, fromage frais (arrivé aussi du Mexique) et de piments $»^{2}$.

23 avril 2005. «Je passe l'après-midi chez Sandra. Le soir, quand son mari revient du travail, ils me proposent de rester pour dîner avec eux. On ne mange pas à la maison, ils m'invitent au McDonald's ». (Extraits du journal de terrain).

6 Cet extrait montre combien la présence d'aliments mexicains dans le régime alimentaire quotidien (tortillas et piments principalement) et la consommation d'aliments acquis pendant l'expérience migratoire (le hamburger) se côtoient. Peut-on parler de la permanence à l'étranger d'une manière de manger qui serait mexicaine même en présence de nouveaux aliments ? Quels sont en réalité les changements que connaît le régime alimentaire des migrants pendant leur installation aux États-Unis? Comment le métissage de ces deux registres culinaires se traduit-il ?

7 L'analyse du lien migration-alimentation à partir de l'opposition ruptures/continuités permet d'identifier aisément la dynamique des abandons et des persistances dans les régimes alimentaires. Cependant, comme le soulignent certains auteurs, cette opposition pour expliquer comment des emprunts faits à un registre culinaire sont interprétés et intégrés dans un autre n'est pas nécessairement heuristique. Aussi, cette vision est-elle insuffisante pour rendre compte des processus de négociation ou de manière plus générale, des relations sociales d'altérité que l'acte alimentaire en situation de migration occasionne (Garnier, 2010 ; Crenn, Hassoun et Medina, 2010). En considérant cette critique, c'est principalement la place du migrant en tant qu'intermédiaire, sorte de traducteur ou de lien entre les pratiques alimentaires de sa société d'origine et celles de la société de destination, que nous voulons ici observer. Dans la mesure où les processus identitaires obligent à penser la relation à l'autre, le fait alimentaire en tant que support de l'identité individuelle et collective peut être également considéré comme un univers changeant, soumis à une élaboration continue, dans laquelle le mangeur se pense lui-même par le biais des autres, selon qu'il partage ou non le même registre culinaire. C'est donc ce rapport à soi et à l'autre à travers les manières de manger que nous étudierons. 


\section{Le terrain et l'enquête}

Notre cas d'étude concerne le village indien de Patamban ${ }^{3}$ (Michoacán, Mexique) et la mobilité de sa population en direction des États-Unis ${ }^{4}$. Les données que nous utilisons ont été recueillies dans le cadre d'une recherche doctorale portant sur la signification de l'espace domestique et le rapport que les sujets entretiennent avec leur double résidence tout en habitant dans un espace migratoire ${ }^{5}$. Suivant la mobilité des sujets, nous avons réalisé un travail d'enquête de type multi-sited (Marcus, 2001) en réalisant des séjours au village, au Mexique, et aussi dans les différents lieux où sont installées les familles de Patamban, aux États-Unis. Dans ce texte, nous nous concentrerons sur les lieux de destination migratoire, sans pour autant négliger le contexte du village d'origine ${ }^{6}$.

Bien que nous n'ayons pas mené une enquête concernant uniquement l'alimentation, l'observation au quotidien des repas pris avec les familles, des courses réalisées dans les magasins de produits mexicains ou dans les supermarchés, des sorties pour aller manger au restaurant ou encore des célébrations auxquelles nous avons assistées, constituent autant de moments à partir desquels nous pouvons fournir une ethnographie des pratiques alimentaires dans le quotidien de ces familles mexicaines vivant aux États-Unis. Nous allons nous servir de ces observations et des extraits des entretiens réalisés avec les migrants pour montrer comment l'expérience de vie migratoire entraîne effectivement l'émergence d'un style alimentaire particulier, composé à la fois d'habitudes alimentaires du pays de départ et d'autres acquises dans la société de destination. Ensuite, nous nous demanderons quel est le sens donné à l'alimentation lorsque le migrant a un régime alimentaire plus proche de son pays d'origine, ou au contraire, plus proche des normes et pratiques de la société d'accueil. Finalement, nous analyserons la manière dont les individus définissent leur identité de migrants à travers l'alimentation.

10 Nous considèrerons d'abord brièvement le contexte de mobilité entre Patamban et les États-Unis pour observer l'organisation de la circulation des aliments entre le village d'origine et les lieux de destination. Nous tiendrons compte aussi de la consommation qui est réalisée dans les commerces de produits mexicains aux États-Unis ce qui nous permettra de visualiser la place des aliments dans la configuration de l'espace migratoire. Ensuite, nous décrirons les différentes manières de manger afin de distinguer comment, où, et avec qui les migrants mangent. Nous serons ainsi en mesure de mieux interroger le lien : migration-alimentation-identité.

\section{La migration de Patamban en direction des États-Unis}

11 Le village de Patamban se trouve dans la Meseta Tarasca, au nord-ouest du Michoacán, une région caractérisée par les anthropologues comme le territoire des Purhépechas, nom également de la langue que parlent les habitants de cette partie du Mexique ${ }^{7}$. En 2010, l'INEGI ${ }^{8}$ évalue la population totale de Patamban à 3602 habitants. Le village est connu dans la région pour sa longue tradition de création de poteries, car le travail dans l'artisanat constituait naguère l'une des principales activités économiques à côté de l'agriculture et du travail du bois. Ces activités ont aujourd'hui disparues au profit du travail en immigration. Pour mieux comprendre les évolutions et continuités du 
phénomène de déplacement vers les États-Unis depuis Patamban, nous distinguerons rapidement trois étapes dans le déroulement du courant migratoire? ${ }^{9}$.

Une première vague de mobilité s'est formée dans les années 1950 et 1960. Elle concernait exclusivement des hommes qui migrent seuls. Cette période correspond au fonctionnement du Programme Bracero (1942-1964), accord signé entre le gouvernement mexicain et celui des États-Unis, avec lequel s'est consolidée la reconnaissance d'un marché de travail binational à partir de l'embauche de travailleurs mexicains, principalement dans le secteur agricole. Au cours des années 1970 et 1980, on a observé une deuxième vague de migration avec la formation de réseaux de circulation au sein des familles. Durant cette période, bien que le programme Bracero ait pris fin, il n'était plus possible de freiner le mouvement des travailleurs patambeños vers le Nord ${ }^{10}$. En s'appuyant sur leur expérience migratoire, les chefs de famille ont commencé à faire venir leurs enfants dans les mêmes lieux de destination qu'eux, donnant naissance à ce qu'on peut considérer comme la consolidation du phénomène de migration ${ }^{11}$. Finalement, une troisième étape s'est dessinée à partir du moment où les femmes et les enfants se sont ajoutés au mouvement migratoire. S'est met ainsi en place une migration familiale qui a conduit à une installation des familles plus stable et de plus longue durée aux États-Unis à partir de la deuxième moitié des années 1990 et principalement au cours des années 2000. Dans ce contexte, pendant les années 1990, des localités précises dans les États de Colorado, Floride et Utah par exemple, sont devenues des points d'ancrage importants qui ont servi de lieux de concentration pour la population patambeña.

Dans le village, on peut distinguer, une norteñización ${ }^{12}$ (Alarcón, 1986) dans le sens où la migration constitue un phénomène partagé, où le savoir-faire est transmis par les migrants aux nouvelles générations, devenant ainsi une pratique quotidienne qui se reproduit continuellement. Les réseaux, pas seulement de circulation des personnes, mais aussi de communication, et les multiples échanges contribuent à donner stabilité et contenu aux relations entre les migrants et leur village, tout en produisant une sorte de simultanéité temporelle qui permet au groupe de maintenir, malgré la dispersion géographique, des liens solides. Tous ces éléments rendent compte de l'existence d'un " champ migratoire international $\aleph^{13}$ dans le sens proposé par le géographe Gildas Simon (1995), au sein duquel des logiques non seulement économiques (bien que la migration soit activée de manière très claire par le manque de travail à Patamban) mais aussi de type symbolique, permettent la structuration de l'espace social international dans lequel circulent et habitent les migrants.

14 Dans le contexte de mobilité que connaît Patamban, les influences qui arrivent du « Nord » sont importantes. Un style de vie plus urbain est repérable, principalement en raison de l'utilisation de la voiture qui a permis un rapprochement avec la ville et favorise l'intégration économique du village dans un réseau plus vaste. La ville est un lieu proposant tous types de produits: vêtements, électroménagers, matériaux de construction, produits pour l'agriculture et l'alimentation. De même, l'accès aux informations par le biais des médias - dont la TV et l'Internet sont les moyens principaux - devient un élément essentiel dans la transformation des habitudes locales, autrefois plus rurales. Les changements vestimentaires témoignent également des mutations dans les styles de vie: l'usage du pantalon chez les femmes (phénomène maintenant très courant) et l'abandon du rebozo ${ }^{14}$ (actuellement moins utilisé surtout chez les jeunes filles). Chez les jeunes garçons, l'adoption de la mode américaine 
consiste à porter des jeans, des baskets et une casquette, ou à arborer le style des cholos $^{15}$ dont la tenue s'inspire des jeunes des gangs de Californie ${ }^{16}$. À l'échelle des maisons, Karine Tinat (2008a) a observé l'acquisition d'appareils électroménagers grâce aux remesas (envois d'argent) que les familles reçoivent des États-Unis, mais qui ne sont pas toujours mis en service car les mères de famille se montrent souvent réfractaires à ces nouveaux biens ${ }^{17}$. Cet auteur propose deux explications à ce phénomène. Tout d'abord, l'achat de ces appareils peut être considéré comme une réponse des familles restées au village en ce qui concerne leur envie de pouvoir « évoluer » au même rythme que les membres de leur famille qui ont migré. Une deuxième piste d'interprétation suggère que, malgré le manque ou la faible utilisation de ces appareils, ces objets représentent un lien émotionnel entre les migrants et ceux qui ne migrent pas (Tinat, 2008c).

Karine Tinat a étudié de manière ponctuelle les pratiques alimentaires des Patambeños en interrogeant l'émergence possible de troubles du comportement alimentaire dans le village, particulièrement parmi les jeunes générations. Ses travaux montrent que « les

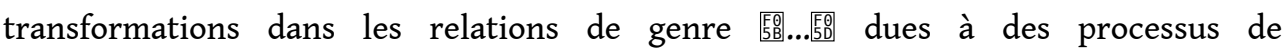
modernisation, peuvent faire émerger des bouleversements des normes alimentaires " (2008b: 664). D'une façon générale, cet auteur considère que «les attitudes alimentaires des familles patambeñas, autant que celles de leurs membres devenus migrants, oscillent entre des mouvements de continuité et de changement » (2008c :19). Les travaux de Karine Tinat ${ }^{18}$ rendent largement compte du cas patambeño. Nous avons poursuivi ses questionnements de l'autre côté de la frontière. Les pistes d'analyse pointées par cette chercheuse ont guidé nos interrogations pour l'observation de l'alimentation des Patambeños dans le contexte américain. Certes, notre travail ne porte pas ici sur les troubles du comportement alimentaire, mais nous insistons surtout sur la nécessité heuristique de penser la dichotomie changements/continuités en considérant les rapports d'altérité auxquels est confronté le migrant. C'est en définissant une plus ou moins grande proximité avec les deux régimes alimentaires qui forment désormais son style alimentaire que le migrant peut définir une manière de manger qui supporte son identité. Car si la migration entraîne des transformations alimentaires à l'intérieur du groupe patambeño, c'est par une synthèse faite de proximité et de distance avec un registre culinaire connu avant la migration que le sujet définit son alimentation en tant que migrant. Pour commencer nous observerons la place des aliments dans les flux de circulation qui soutiennent le champ migratoire patambeño.

\section{La circulation des aliments dans l'espace migratoire}

Pour comprendre la configuration de l'espace migratoire patambeño, il est nécessaire de considérer les flux qui existent entre les différents points de l'espace migratoire, car comme le suggère Laurent Faret, «si les réseaux sociaux sont les structures du système migratoire, les flux qui les parcourent sont les lignes de force du champ qui s'établit entre les différents pôles de ce système " $(2003: 224)$. Suivant cette lecture, on peut distinguer que les biens qui circulent le plus dans le sens Nord-Sud sont l'argent et les cadeaux. En revanche, les aliments ont une place centrale dans la circulation qui va du Sud vers le Nord. 
17 La circulation des produits comestibles s'opère à travers la circulation des personnes. Ce sont les migrants « qui ont des papiers » et qui reviennent au moins une fois par an au village, qui constituent les principaux intermédiaires de ce type de flux. Au moment de retourner au Nord, ils remplissent leurs voitures de produits comestibles qu'ils distribueront ou partageront aux États-Unis avec les membres de leur famille ou amis du village. De même, depuis Patamban les mères de famille envoient des colis postaux avec des produits locaux que les migrants sont censés ne pas pouvoir acquérir aux États-Unis. Un épisode vécu pendant l'enquête de terrain rend bien compte de cette dynamique :

Patamban, mars 2007. « Rosa me propose de venir la voir chez elle en Californie. Avant mon voyage, ses parents me demandent si je peux lui emporter «quelques trucs ». La veille de mon départ, je vais chez eux et une grosse valise remplie exclusivement de nourriture m'attend. Une grande quantité de pains, des morceaux de viande sèche, quatre poissons secs, des piments, une sorte de compote préparée à base de citrouille et de canne à sucre que les Patambeños nomment conserva dans un grand seau en plastique et, finalement, quelques herbes pour des remèdes médicinaux remplissent la valise. Une fois chez Rosa, nous découvrons que la conserva est avariée et Rosa doit la mettre directement à la poubelle. Il ne me semble pas qu'elle soit déçue. Les herbes médicinales ont trop séché pendant le voyage et elle s'en débarrasse aussi immédiatement. C'est le pain qui parait avoir le plus de succès. Le soir même, tous les membres de la famille le mangent accompagné d'un verre de lait frais. Lors de mon retour au village, Rosa me redonne la même valise, mais cette fois remplie de vêtements et autres cadeaux pour sa famille ».

18 Comme nous l'avons indiqué, dans la configuration des flux de circulation, l'argent vient du Nord. C'est aussi dans ce sens que l'accès à la consommation subit des modifications. Postes de télévision, chaînes Hi-Fi, appareils photos, fours micro-ondes, vêtements, téléphones portables et, en général, toutes sortes de cadeaux sont envoyés par les migrants à leurs familles restées au village. En sens inverse, des poteries, des habits typiques utilisés pour les danses traditionnelles lors des célébrations religieuses, mais surtout des aliments sont envoyés depuis le village. Dans une logique d'échange, les aliments locaux sont pour ceux qui ne partent pas une manière de "donner " quelque chose aux migrants. L'envoi d'aliments depuis le village se présente clairement comme une preuve d'affection de la part de ceux qui restent envers ceux qui partent. Cela permet de nourrir des liens dans la distance. Ce type de pratique a été relevé dans le cas d'autres migrations. A propos des Camerounais installés en région parisienne, Bouly de Lesdain a observé que «l'envoi et la réception de denrées matérialisent un réseau qui lie les migrants à l'espace d'origine et les migrants entre eux » (2002:10). Dans la migration des Mixtèques originaires de la région d'Oaxaca, Françoise Lestage a également identifié que les dons alimentaires réaffirment des relations familiales qui pourraient perdre de leur force à cause de l'éloignement (2008b).

Par ailleurs, on peut aussi considérer que les aliments fonctionnent comme une force de rappel : éviter que les migrants oublient certains goûts de la cuisine de leur pays, stimulant en même temps un contact émotionnel avec l'ensemble de leur culture d'origine. Les aliments servent à éveiller une mémoire gastronomique commune. Objet d'identification au même groupe, la conserva est par exemple un plat qui est préparé et consommé seulement pendant les célébrations qui accompagnent la Semana Santa (pâques). Recevoir ce plat aux États-Unis permet en quelque sorte de revivre le temps rituel qui est vécu au village. Ainsi, les aliments créent du lien d'abord parce qu'ils sont 
partagés entre les membres de la famille, mais aussi au-delà parce qu'ils confirment une connaissance des uns et des autres par le biais de la connaissance des mêmes aliments. On pourrait jouer sur les mots et dire que la circulation des aliments entre le village et les États-Unis permet de «nourrir » des proximités tout en consolidant des liens (familiaux et amicaux), en même temps que ces aliments aident à "digérer » les distances. C'est par le partage et la consommation d'un aliment commun que, malgré l'éclatement géographique qui les sépare, les patambeños mangent ensemble.

\section{Les commerces de produits mexicains aux États-Unis} peut distinguer la présence de commerces dans lesquels des produits d'origine mexicaine peuvent être acquis. Ce type de commerce est courant aux États-Unis. Ceci s'explique en partie par la hausse démographique de la population hispanique et notamment mexicaine ${ }^{19}$. La forte demande des produits mexicains contribue à l'essor de ces magasins tout en permettant de maintenir les traditions culinaires du Mexique aux États-Unis.

21 L'anthropologue, Shinji Hirai, qui travaille sur la mobilité des Mexicains originaires de la région du Jalisco en direction des États-Unis, s'est penché sur le fonctionnement de ce type de magasin. Dans son étude, il forge la notion de "marché de la nostalgie ", à partir de laquelle il cherche à définir le lien qui existe entre l'ouverture d'un commerce avec des produits mexicains comme activité économique réalisée par un migrant et l'offre de produits qui sert d'identification à leur culture. Pour cette chercheuse, les commerces proposent des produits mexicains pour stimuler la nostalgie du pays. La consommation de ces produits serait alors un acte d'identification permettant de ratifier une certaine manière "d'être mexicain" qui, tout en recréant des liens émotionnels avec le lieu d'origine, contribuerait à la définition d'un sentiment d'appartenance collective (Hirai, 2009).

Pour sa part, Françoise Lestage s'est intéressée à ce type de commerce chez les indigènes d'origine mixtèque de la région de l'Oaxaca (Mexique) qui migrent, soit dans la ville de Tijuana, soit dans les différentes villes californiennes aux États-Unis. Lestage fait la distinction entre deux types de circuits marchands : celui qui est destiné à une demande locale, et celui qui, au contraire, vise aussi une demande extérieure. Le premier se réfère aux produits vendus dans un espace social uniquement mixtèque; le second correspond à la vente à des clients extérieurs au groupe identitaire de celui qui tient le commerce (Lestage, 2008a). C'est le premier cas qui nous intéresse ici, car pour le moment, les Patambeños ne sont pas eux-mêmes propriétaires d'un commerce de ce type. Mais par contre, ils consomment des produits alimentaires dans des magasins tenus par des personnes d'origine mexicaine.

À cet égard, nous distinguons deux types de magasins de produits mexicains fréquentés par les Patambeños. Le premier correspond à des établissements de type "épicerie " consacrés uniquement à la vente de produits comestibles tels que boîtes de conserve de différentes sortes de piments, grains de maïs pour préparer le pozole, tortillas et tostadas, feuilles de maïs pour la préparation des tamales, fromage frais, etc. On y trouve aussi des boissons de marques mexicaines, des bonbons (à base de sucre et piment) et encore des cacahuètes, des fèves ou des pois chiches secs pimentés. Cette présentation des produits destinés au grignotage est très courante au Mexique. Le mode de 
consommation dans ces établissements est apparenté à la fonction que détient "l'épicerie du coin" dans le village d'origine. Il s'agit donc d'un commerce de proximité dans lequel les gens peuvent se rendre plusieurs fois par jour pour acheter à chaque fois seulement un ou deux produits pour compléter la préparation du repas du jour : quelques citrons, des avocats, des œufs, une bouteille d'huile, un soda, etc.

Dans le deuxième cas, il s'agit de commerces plus importants qui s'apparentent davantage aux supermarchés. Leur offre de produits est pratiquement similaire au type de commerces précédents, mais ils proposent en plus un service de boucherie et de boulangerie. Les ménages avec lesquels nous avons réalisé des achats dans ce deuxième type de commerce déclarent toujours qu'ils préfèrent acheter la viande dans ces endroits, car celle-ci est exposée et coupée directement selon la commande du client. Les migrants sont en effet réticents à l'achat de viande en sachet, fidèles en ce sens aux habitudes de leur village, puisque la viande y est toujours achetée le jour même de l'abattage de la bête. Dans ces établissements, les ménages réalisent des courses de plus grande importance, prévoyant des aliments pour une semaine par exemple.

Le succès de ces magasins vient du fait qu'outre la vente des produits, ils offrent un service de restauration de plats mexicains à consommer sur place ou à emporter. Des $\operatorname{tacos}^{20}$ avec différents types de garnitures, des tortas, quesadillas, tamales et du pozole, etc. y sont servis. Dans tous les lieux que nous avons connus, les femmes qui préparaient les plats dans les cuisines étaient mexicaines. Ce service est principalement utilisé par les jeunes célibataires qui ont migré sans leur famille, qui habitent dans des colocations exclusivement masculines et qui mangent la plupart du temps hors de leur domicile. Ces lieux sont plus informels que les restaurants et leur permet de " manger mexicain » à peu de frais. Le fait de pouvoir y parler espagnol est vraisemblablement un facteur important qui contribue aussi à attirer la clientèle.

26 La possibilité de se fournir facilement dans ces commerces en ingrédients nécessaires à la préparation des plats mexicains pourrait laisser penser que l'envoi d'aliments depuis le village d'origine n'a pas de sens. Cependant, nous avons noté que ce qui importe le plus dans la cuisine réalisée avec des produits arrivés de Patamban, est la notion de partage : l'échange d'aliments permet de renforcer des liens d'appartenance avec les membres de la famille et dans un sens plus large avec les gens du village. Parallèlement, la consommation aux États-Unis permet avant tout au migrant de distinguer sa manière de manger du reste de la société dans laquelle il vit. La fréquentation de ces commerces traduit donc le rattachement du migrant à ses compatriotes, marquant dans le même temps sa différence avec les autres groupes de la société d'accueil. Ces espaces favorisent ainsi une forme de socialisation qui passe par un rapport commun aux aliments, support de l'émergence d'une spécificité identitaire à partir de l'alimentation.

Nous avons souligné la centralité des aliments dans la configuration des liens d'appartenance avec le village d'origine et l'importance des commerces de produits mexicains comme espaces d'identification à la culture mexicaine. Nous allons à présent décrire la composition des repas pris au quotidien afin de distinguer quelles sont les pénétrations observables entre les registres culinaires mexicains et étatsuniens. L'identification de persistances ou d'abandons d'une cuisine d'un côté et l'adoption de nouvelles habitudes alimentaires de l'autre nous permettra de comprendre la place qu'accordent les migrants à l'un ou à l'autre registre et d'analyser comment cette recomposition leur permet de fonder une spécificité alimentaire servant de support à leur identité de migrant. 


\section{Manger hors du pays, dans le nouveau chez soi}

Comme au village, la préparation des repas aux États-Unis est assurée presque exclusivement par les femmes. Par contre, la majorité des hommes qui migrent seuls préfèrent manger hors de leur domicile - comme on l'a vu dans les magasins mexicains - ou bien en achetant des produits déjà préparés et prêts à être réchauffés au four à micro-ondes. Dans les maisons, les prises alimentaires suivent la temporalité des journées de travail. Très tôt le matin - entre 5 et 6 heures - les hommes partent travailler sans prendre de petit déjeuner. Vers midi, ils prennent le lonche ${ }^{21}$ que leurs femmes ont préparé la veille - sorte de casse-croûte qu'ils «cassent » pendant leur pause-déjeuner - et qui est le plus souvent composé d'œufs, d'haricots noirs et de piments, le tout accompagné de tortillas. Quand ils n'ont pas de lonche faits à la maison, ils achètent des sandwichs et un soda dans les commerces à proximité de leur lieu de travail. Le repas le plus important est celui qui a lieu après le travail, vers 15 heures, quand les hommes reviennent à la maison. Ce repas est toujours accompagné de tortillas de maïs que les femmes préparent elles-mêmes chaque jour - comme il est habituel de le faire au village -, ou ont acheté en sachet dans les magasins et supermarchés. Les frijoles (haricots) sont également presque toujours présents. Les plats qui composent ce repas se retrouvent dans toute la cuisine mexicaine : chiles rellenos, enchiladas, caldo de pollo, tortas, tostadas, rajas con huevo, chilaquiles, etc. La consommation de sodas est surprenante et peut s'élever à trois ou quatre canettes par jour et par personne. Les migrants s'en servent souvent comme critère pour définir leur nouvelle façon de consommer. Plusieurs fois les migrants nous ont parlé du bonheur de pouvoir servir un soda à chaque membre de la famille à chaque repas, en se souvenant du temps où ils devaient partager une bouteille de deux litres avec toute la famille. Le soda se présente clairement comme un signe d'opulence économique conquis grâce à la migration.

En effet, les habitudes alimentaires sont souvent utilisées comme repères pour comparer le mode de vie aux États-Unis par rapport à celui du village d'origine. Le propos de cette femme est révélateur de ce mode de raisonnement: « Les différences entre là-bas et ici, c'est d'abord la nourriture. Ici, c'est très différent, c'est pour ça que les gens changent. [...]. Aux enfants, on leur donne l'habitude de ne manger que des hamburgers... et alors, la nourriture, ils ne la mangent plus. Ils ne boivent plus d'atole ${ }^{22}$, ils ne boivent plus de lait, juste des hamburgers et des céréales, c'est ça qu'ils mangent $»^{23}$.

Les hamburgers et les céréales apparaissent comme n'étant pas une " vraie » nourriture aux yeux de cette femme, elle n'en mange pas, mais sait que les enfants en mangent. Au village, un grand-père nous a parlé de l'alimentation de son petit-fils né aux États-Unis en nous disant que celui-ci ne voulait pas manger de tortillas : «Le gamin était habitué à ne manger que du pain, alors je lui ai dit : écoute, ici tu vas manger les plats que ta grand-mère prépare, ici ce n'est pas un restaurant. Il mangeait alors avec la cuillère toute la nourriture mais sans manger de tortillas. Après, il avait vite faim! Je lui ai expliqué que c'est parce qu'il ne mange pas de tortillas qu'il a faim. Il faut qu'il mange des tortillas ${ }^{24}$. Ces exemples nous montrent l'importance de la socialisation dans la construction des valeurs et usages associés aux aliments mais on peut constater également qu'il existe un décalage générationnel dans l'adoption de nouveaux plats. D'autres situations nous ont permis de le constater lors de repas en famille où les 
parents mangeaient un caldo de pescado (bouillon piquant préparé à base de poisson sec et de légumes) et avaient commandé une pizza pour les enfants. Dans un autre cas, les enfants mangeaient des cuisses de poulet surgelées et réchauffées au four micro-ondes tandis que les parents mangeaient des chiles rellenos (poivrons farcis). Les parents nous ont expliqué que leurs enfants nés aux États-Unis n'aiment pas la nourriture mexicaine. La nouvelle génération de Patambeños née aux États-Unis est certainement plus prédisposée à acquérir, au contact de ses pairs, des habitudes alimentaires de la société d'accueil, principalement par le biais de la socialisation à travers l'école. On peut considérer qu'à partir d'un phénomène d'imitation, elle contribue à modeler de nouvelles pratiques alimentaires au sein de ces familles. Les enfants, liés au sol étatsunien par naissance, seraient plus « légitimes » à manger des plats américains. Ils acquièrent dès lors le rôle d'intermédiaires culinaires dans l'intégration de nouveaux aliments au régime alimentaire des familles patambeñas. Il nous semble que dans l'équation : naître aux États-Unis = aimer les plats américains, les familles patambeñas cherchent à trouver un canal d'introduction des nouveaux aliments dans leur système culinaire. Ici, les enfants deviennent un lien, un espace de découverte et de contact avec de nouveaux goûts. On peut dire que « si l'acte alimentaire insère et maintient par ses répétitions quotidiennes le mangeur dans un système de significations " (Poulain, 2002 : 177), c'est à travers les enfants que semble s'opérer l'apprentissage d'une nouvelle cuisine.

\section{Aller au restaurant}

31 Lié à la condition économique des familles, car " plus le niveau de vie s'accroît, plus la cuisine sort de la sphère domestique » (Fumey, 2007 : 78), le choix de la fréquentation des restaurants obéit cependant à d'autres logiques que celles purement monétaire. La majorité des personnes qui nous ont proposé d'aller manger au restaurant en ont choisi un de type mexicain. Dans ce type d'établissement, les plats ressemblent à ceux qui sont offerts dans les services de restauration des magasins dont nous avons parlé plus haut (des tacos, des tortas, du pozole, des enchiladas sont offerts dans les menus). Dans ces endroits, autant le service que les clients sont d'origine mexicaine, et le fait de pouvoir parler espagnol, semblent être des éléments importants qui déterminent la fréquentation de ces lieux. Deux exemples nous permettront de valider cette observation.

Floride, mai 2005. Ce soir Luis et Daniel me proposent de dîner avec eux. On va dans un magasin-restaurant mexicain où, disent-ils, on peut manger. Il est 22 heures et ce lieu est fermé. On essaye cette fois-ci un vrai restaurant mexicain, mais encore sans succès. Ils décident de tenter le Burger King. Là-bas, on a juste à choisir un menu avec un numéro. Car, « à cette heure-ci, il y a d'autres endroits qui servent à manger, mais il faut savoir parler anglais ».

Colorado, juin 2005. Roberto et sa femme Rosa m'invitent à manger. Nous allons dans un restaurant chinois. Je suis surprise d'aller manger autre chose que de la nourriture mexicaine. Dans ce restaurant, on passe directement à la caisse pour payer un prix unique pour chaque personne. Roberto dit three, tout en faisant un signe avec ses doigts. Il paie trois menus et en échange obtient un ticket avec lequel chacun de nous prend un plateau et des couverts. On peut ensuite se servir autant de fois qu'on veut des différents plats disponibles au buffet qui est proposé.

En dehors du besoin de parler anglais comme critère définissant le type de restaurant que les migrants vont fréquenter, nous aimerions souligner le fait que «les 
modifications des comportements alimentaires (peuvent être fortement liées) à des processus comme l'urbanisation, la tertiarisation, la hausse du niveau de vie » (Fumey, 2007 : 77). Dans le village d'origine, il n'y a aucun restaurant et la fréquentation des lieux de restauration est directement liée à la condition migratoire. Beaucoup de migrants nous ont parlé de cette condition en la comparant toujours avec leur lieu d'origine. "À Patamban, il y a beaucoup de misère, ici (aux États-Unis) tu peux te donner le plaisir d'aller au restaurant, de goûter des bonnes choses. Tu peux aller au supermarché et t'acheter des choses que tu aimes. Au village, même pour manger un morceau de viande, c'est difficile $»^{25}$. Dans ce contexte, aller au restaurant se présente comme un critère important dans la définition d'un nouveau style de vie et comme moyen d'appropriation de l'espace où ils habitent à présent. Par le biais du restaurant, les migrants tissent un rapport avec l'espace qu'ils habitent dans la mesure où le restaurant constitue un lieu où il est possible de rencontrer d'autres Mexicains. «En recomposant leurs liens à l'espace et aux territoires (où ils habitent), les migrants recomposent forcément leurs manières d'intégrer ces territoires-là, au plus intime d'eux-mêmes" ici, à travers des pratiques alimentaires (Fumey, 2007: 82). La fréquentation de restaurants permet aux migrants de consommer des aliments dans un cadre nouveau qui est aussi un espace de socialisation soutenant les processus de transformation identitaire qu'ils vivent. Dans ce sens, il ne s'agit pas de considérer la consommation d'aliments et de plats mexicains comme des traits culturels fixes qui serviraient à définir la permanence d'un régime alimentaire acquis dans le village d'origine, mais au contraire de montrer qu'avec l'accomplissement de pratiques culinaires semblables à celles du pays d'origine, mais réalisées dans un contexte étranger, les migrants utilisent les aliments comme des référents pour distinguer leur culture d'origine des emprunts qu'ils font à la culture dans laquelle ils habitent désormais.

\section{Conclusion : manger comme un migrant}

33 Combien de fois les migrants nous ont-ils parlé de leur nostalgie de la nourriture mexicaine, ou plus précisément, des plats régionaux ou locaux. «Ce qui me manque le plus de Patamban, ce sont ses repas: le churipo ${ }^{26}$, les tamales $^{27}$, les corundas $^{28}$, les huchepos. (Mais ici tu peux manger tout ça, non ?) Oui, mais ce n'est pas pareil, ça n'a pas le même goût, même quand c'est quelqu'un de Patamban qui le prépare, ce n'est pas pareil. Je ne sais pas si ce sont les marmites, ou bien le feu de bois $»^{29}$. Ou alors, « ici, l'inconvénient c'est que tu n'es pas dans ton pays, tu ne peux pas manger tes charibolas, tes enchiladas, tes tamales. (J'ai l'impression qu'ici on peut manger la même chose que là-bas, non ?) Enfin, oui, mais il manque l'essence, le goût de la nourriture, il y a quelque chose qui fait que ce n'est pas pareil $»^{30}$. Quelle est cette essence qui manque? Quel est, d'un autre point de vue, ce sentiment qui donne sens à l'appartenance à travers les aliments ? En effet, on peut croire que la préparation des aliments dans des marmites en terre cuite et au feu de bois, comme il est courant de le faire au village, donne un certain goût aux plats, goût qui serait altéré ou transformé dans les cuisines aux États-Unis. Cependant, il nous semble que plus que le goût « réel » des aliments, les discours des migrants servent à marquer leur appartenance d'origine, les aliments appartiennent au migrant parce qu'ils sont associés à l'espace d'origine, ils marquent dans ce sens l'appartenance du migrant à son groupe. Les aliments se retrouvent désormais faisant partie du répertoire des souvenirs à travers lesquels les migrants 
élaborent une mémoire commune, toujours marquée par la rupture, par le manque, par la nostalgie. Inversement, on a observé qu'avec l'adoption d'autres aliments, les migrants cherchent à définir une manière de manger qui serait plus moderne, principalement parce qu'elle est différente de la manière dont la plupart ont mangé dans le passé. Nous avons relevé en particulier que la consommation d'aliments surgelés ou proposés par des chaînes de fast food par les enfants s'apparentait à un canal de diffusion de nouveaux aliments dans l'espace domestique des familles migrantes. Nous pouvons mentionner encore un exemple amusant, mais néanmoins révélateur de la mise en scène de l'alimentation dans le contexte de la migration. En 2011, un groupe a été créé, sur le réseau social facebook, sous le nom de Patambeños en la web : les migrants et non-migrants y échangent, entre autres, des photos de nourriture. Il s'agit soit de repas «traditionnels » que les gens préparent chez eux, aux États-Unis, soit de plats pris lors de repas au restaurant. L'échange de ces photos de plats semble ainsi assurer un double rôle pour ces personnes : d'une part, montrer qu'elles continuent à manger " à la mexicaine » légitimant ainsi leur identité d'origine, d'autre part, montrer aux membres de leur groupe leur intégration dans la société de destination par la fréquentation de restaurant.

S'il est préférable de sortir de la dichotomie continuité/discontinuité du système alimentaire, c'est parce que l'enjeu vécu par les migrants ne se pose pas en termes d'opposition entre conserver ou transformer une manière de manger. Tout d'abord, l'étude de la préparation des repas montre la permanence des goûts dans l'alimentation quotidienne confirmant une certaine stabilité de l'alimentation familiale du pays d'origine. Le lien que les migrants entretiennent avec leur village, transformé en raison de la mobilité migratoire, vient doter les aliments du pays d'une dimension symbolique supplémentaire qui est celle de l'aliment comme identificateur, signe d'un attachement affectif à leur culture d'origine. Par ailleurs, ce processus n'empêchera pas les familles patambeñas d'intégrer de nouveaux goûts dans leur alimentation. Avec le passage d'un pays à un autre, les migrants deviennent les créateurs d'espaces qui servent de passerelle entre les modèles alimentaires. Avec la mobilité, les goûts se mélangent et le registre identitaire des migrants se crée et se renouvelle à travers une nouvelle manière de manger qui obéit aux nouvelles règles d'alimentation plus conformes aux normes du pays d'origine ou bien, à celle du pays d'accueil.

\section{BIBLIOGRAPHIE}

ALARCÓN Rafael, «El proceso de norteñización : impacto de la migración internacional en Chavinda, Michoacan » in Thomas CALVO et Gustavo LÓPEZ (eds), Movimientos de población en el occidente de México, México, COLMICH-CEMCA, 1986, pp. 337-357.

BAHLOUL Joëlle, La maison de mémoire. Ethnologie d'une demeure judéo-arabe en Algérie (1937-1961), Paris, Métaillé, 1992.

BODY-GENDROT Sophie, Les États-Unis et leurs immigrants. Des modes d'insertion variés, Paris, Études de La Documentation Française, 1991. 
BOULY de LESDAIN Sophie, « Alimentation et migration, une définition spatiale » in Isabelle GARABUAU-MOUSSAOUI, Élise PALOMARES et Dominique DESJEUX (eds), Alimentations contemporaines, Paris, L'Harmattan, 2002.

CRENN Chantal, HASSOUN Jean-Pierre et MEDINA François-Xavier, « Introduction : Repenser et réimaginer l'acte alimentaire en situations de migration ", Anthropology of food, n 7, 2010. Disponible en ligne : http://aof.revues.org/6672

CERTEAU de Michel, GIARD Luce et MAYOL Pierre, L'invention du quotidien, t. 2. Habiter, cuisiner, Paris, Gallimard, coll. « Folio Essais », 1994.

DURAND Jorge et ARIAS Patricia, La vida en el Norte. Historia e iconografía de la migración MéxicoEstados Unidos, Guadalajara, Universidad de Guadalajara, 2004.

FARET Laurent, Les territoires de la mobilité. Migrations et communautés transnationales entre le Mexique et les États-Unis, Paris, CNRS, 2003.

FUMEY Gilles, « La mondialisation de l'alimentation », L'Information géographique, vol. 71, $\mathrm{n}^{\circ} 2$, 2007, pp. 71-82. Disponible en ligne : www.cairn.info/revue-l-information-geographique-2007-2page-71.htm.

FISCHLER Claude, L'Homnivore, Paris, Odile Jacob, 2001.

GARCÍA-GARZA Domingo, « La redéfinition des plats et des pratiques alimentaires populaires au Mexique ", IdeAs, $\mathrm{n}^{\circ}$ 3, 2012. Disponible en ligne : http://ideas.revues.org/461.

GARCÍA-GARZA Domingo, « Una etnografía de los tacos callejeros en México. El caso de Monterrey », Estudios sociales, vol. 19, n³7, 2011, pp. 32-63.

GARCÍA-GARZA Domingo, « Prácticas alimenticias y clasificación social. ¿Los tacos son un alimento popular?», Civitas, vol.10, n 3, 2010, pp. 430-449.

GARNIER Julie, «'Faire avec' les goûts des autres », Anthropology of food, n 7, 2010. Disponible en ligne : http://aof.revues.org/6576.

GHORRA-GOBIN Cyntia, «L'entrée des Latinos sur la scène métropolitaine américaine : une ambivalence marquée par des tensions politiques et une influence certaine sur le désir de centralité ", Cahiers des Amériques Latines, vol. 3, n 59, 2008, pp. 123-144.

HASSOUN Jean-Pierre, « Deux restaurants à New York : l'un franco-maghrébin, l'autre africain », Anthropology of food, $\mathrm{n}^{\circ}$ 7, 2010. Disponible en ligne : http://aof.revues.org/6730.

HIRAI Shinji, Economía política de la nostalgia. Un estudio sobre la transformación del paisaje urbano en la migración transnacional entre México y Estados Unidos, México, Universidad Autonoma Metropolitana/Juan Pablos Editor, 2009.

KATZ Esther. « Les normes alimentaires des paysans mixtèques (État d'Oaxaca, Mexique) », Journal des Anthropologues, $\mathrm{n}^{\circ}$ 106-107, 2006, pp. 205-223. Disponible en ligne: http:// jda.revues.org/1330.

LESTAGE Françoise, Les indiens Mixtèques dans les Californies contemporaines. Migrations et identités collectives, Paris, PUF, 2008a.

LESTAGE Françoise, « De la circulation des nourritures. La perpétuation et l'extension des liens sociaux des migrants mexicains via l'approvisionnement en produits alimentaires ", Anthropology of food, 2008b, Disponible en ligne : http://aof.revues.org/index2942.html.

MARCUS George, «Etnografía en/del sistema mundo. El surgimiento de la etnografía multilocal », Alteridades, $\mathrm{n}^{\circ}$ 22, 2001, pp. 111-127. 
POULAIN Jean-Pierre, Sociologies de l'alimentation. Les mangeurs et l'espace social alimentaire, Paris, PUF, 2002.

ROZIN Elisabeth, The Flavouring-Principle Cookbook, New York, Hawthorn, 1973.

SIMON Gildas, Géodynamique des migrations internationales dans le monde, Paris, PUF, 1995.

SIMON Gildas, « Migrations, la spatialisation du regard », REMI, vol. 22, n², 2006, pp. 9-21.

Disponible en ligne: http://remi.revues.org/2815

TINAT Karine, « Les troubles alimentaires émergent-ils en milieu rural ? Regards croisés entre une approche médicale « occidentale » et les représentations et pratiques alimentaires et corporelles de jeunes d'un village de Michoacán (Mexique) », Anthropology of food, May-2008a. Disponible en ligne: http://aof.revues.org/index3853.html

TINAT Karine, « $i$ Existen la 'anorexia’ y la 'bulimia' en el medio rural ? Nuevas representaciones y prácticas alimentarias y corporales entre los jóvenes de Patamban, Michoacán », Estudios sociológicos, vol. 26, n³ 2008b, pp. 647-667.

TINAT Karine, « ¿Y qué pasa con los que se quedan? Del mercado a la mesa : el impacto de la migración en la alimentación (Patamban, Michoacán) » in José Luis SEEFOO (ed.), Desde los colores del maíz. Una agenda para el campo mexicano, t. 2, Zamora, El Colegio de Michoacán, 2008c, pp. 1-24.

\section{NOTES}

1. La fina est un pain typique de Patamban qui se distingue des autres, parce qu'il contient des morceaux de piloncillo (pain de sucre).

2. Au village, un repas ordinaire est aussi constitué de maïs, d'haricots et de piment. Ces trois aliments sont la signature identitaire de la cuisine nationale. Il s'agit en réalité d'une combinaison qu'on retrouve dans le régime alimentaire de tous les paysans méso-américains (Katz, 2006). Aussi, Katz explique que la saveur pimentée est en effet, pour la majorité des Mexicains, ce que Rozin (1973) appelle un flavouring principle, une " saveur de base " sans laquelle on ne reconnaît pas ses aliments.

3. Patamban se situe au nord-ouest du Michoacán, dans la partie occidentale du Mexique. Appartenant à la zone connue comme La Meseta Tarasca, ses habitants ont néanmoins abandonné l'usage du Tarasco qui est la langue indienne parlée dans cette partie du Mexique. Village rural d'implantation franciscaine, fondé aux alentours de 1524, celui-ci s'étend sur 20000 hectares de propriété communale, à une altitude de 2140 mètres.

4. Nos enquêtes concernent trois lieux de destination : la localité de Pueblo dans le Colorado, Fort Pierce en Floride et St George dans l'Utah. Ces lieux sont les ancrages les plus importants en raison du nombre de familles regroupées. Ils offrent par ailleurs le plus fort dynamisme dans le processus contemporain de mobilité entre Patamban et les États-Unis.

5. L'intitulé exact de ce travail de recherche est: La maison du migrant. Mobilités et identités à Patamban (Michoacán, Mexique), thèse en anthropologie soutenue à l'EHESS de Paris le 9 mai 2012 sous la direction d'Alain Musset.

6. Nous avons rencontré au total 79 familles dans l'ensemble des localités de destination où nous avons réalisé notre enquête et avons habité chez 6 familles différentes.

7. La langue purhépecha n'est plus utilisée à Patamban, elle est au contraire en voie de disparition. Seule l'une des trois écoles primaires l'a incorporée à son programme scolaire depuis l'an 2000. Au village tout le monde communique en espagnol. 
8. L'Instituto Nacional de Estadística, Geografía e Informática (INEGI) est l'institution publique chargée des recensements et statistiques nationales au Mexique dont l'INSEE est l'équivalent en France.

9. Celui-ci peut être considéré comme un modèle du fonctionnement migratoire depuis les régions rurales du Mexique.

10. Le «Nord» est la manière courante de se référer aux États-Unis à cause de sa position géographique au nord du Mexique.

11. La Loi IRCA (Amnistie de 1986, aussi connu sous le nom de Loi «Simpson-Rodino» selon le nom de ses auteurs) marque un tournant important dans cette deuxième période du mouvement puisque les migrants qui jouissent maintenant davantage d'expérience vont profiter du contexte politique pour régulariser le statut de résidence de certains membres de leur famille. Cette Loi a permis la légalisation des clandestins ayant résidé sans interruption aux États-Unis depuis janvier 1982 et a permis une amnistie pour les travailleurs agricoles sans papiers qui avaient été employés pendant au moins 90 jours au cours des 12 mois précédents le $1^{\mathrm{er}}$ mai 1986.

12. Dans les régions où il existe des taux importants de migration et une continuité historique du phénomène, l'apparition de réseaux familiaux permet de parler de l'existence d'une « culture migratoire $»$.

13. Cet auteur a d'abord proposé, en 1976, le concept d'espace transnational pour considérer les espaces que les individus parcourent en réalisant des mobilités entre deux ou plusieurs pays. Par la suite, il privilégiera en 1979 la notion de «champ migratoire international » : selon lui, le concept de champ rend mieux compte des forces symboliques qui façonnent le fonctionnement des relations qui ont lieu dans les espaces de la mobilité. Pour plus de détails, voir Gildas Simon (2006).

14. Châle en laine que les femmes utilisent traditionnellement dans l'espace public. Dans le cas de Patamban, le rebozo typique est à rayures bleues et noires.

15. Des pantalons larges qui se portent au-dessous de la taille, la tête rasée et des tatouages constituent les éléments qui caractérisent cette tenue.

16. Dans les quartiers des villes américaines où les Mexicains décident d'installer durablement leur résidence, l'apparition de personnages typiques caractérisés par leurs façons particulières de s'habiller, de se coiffer et de se comporter a servi à marquer les différences ethniques et culturelles avec d'autres populations. Il y a eu le pachuco dans les années 1940, le slow rider des années 1970, et le cholo des années 1980 (Arias et Durand, $2004: 220$ ).

17. Dans son travail, Karine Tinat note que selon les données de l'INEGI (le système de statistique mexicain) de l'an 2000, sur les 782 maisons recensées à Patamban, 199 disposaient d'un réfrigérateur. Aucun renseignement n'est fourni quant à la possession d'une gazinière (Tinat, 2008a). Le recensement de 2010 indique que 463 maisons disposent d'un réfrigérateur.

18. Nous renvoyons le lecteur désireux d'en savoir davantage sur les pratiques alimentaires à Patamban et ses transformations, aux travaux de Karine Tinat qui a largement étudié toutes ces questions.

19. Les Mexicains constituent la population la plus nombreuse au sein de la minorité hispanique aux États-Unis. Sur une population de 44,2 millions de personnes d'origine hispanique, $64 \%$ sont mexicains, soit 28,3 millions (Ghorra-Gobin, 2009).

20. Le taco constitue l'une des principales formes d'alimentation au Mexique. Il s'agit d'une tortilla (galette de maïs) roulée et farcie avec différents types d'aliments. Sur le taco, en tant qu'aliment populaire et « plat national » au Mexique, voir Garcia-Garza (2010, 2011, 2012).

21. Le mot lonche couramment utilisé au Mexique pour désigner un sandwich vient du mot anglais lunch.

22. L'atole est une bouillie de maïs, parfois combinée avec du lait. Il existe une grande variété d'atoles qui peuvent être salés et parfumés au piment ou bien sucrés à base de fruits (à la fraise, au tamarin, à la goyave). 
23. Entretien n. 1950 à Fort Pierce (Floride, EUA) au moi de mai 2005 avec Esmeralda. Migrante depuis 1996.

24. Entretien n. 1926 à Patamban (Mexique) au mois de janvier 2006 avec Armando. Ex-migrant de retour à Patamban.

25. Entretien n. 1969 à St George, Utah (EUA) au mois de mars 2007. Francisco, migrant depuis 1984. .

26. Le churipo est un bouillon piquant avec de la viande de bœuf et des légumes. Il est consommé à Patamban lors des fêtes d'anniversaire, des baptêmes, etc.

27. Les tamales sont un aliment préparé à base de maïs moulu et de saindoux dont on forme une pâte qui est farcie à la viande de poulet ou de porc puis baignée avec une sauce pimentée et cuite à la vapeur enveloppée dans une feuille de maïs.

28. Les corundas sont une variété de tamal typique de la région du Michoacán. Leur caractéristique est que le maïs est lavé dans des cendres de chêne puis enveloppé dans les feuilles de la plante du maïs (et non pas dans celles de l'épi comme pour les autres types de tamales). Elles sont farcies de sauce piquante et parfois de fromage. Les huchepos sont des tamales sucrés, consommés principalement pour le petit déjeuner.

29. Entretien n. 1969 à Fort Pierce (Floride, EUA) au mois de mai 2005. Raúl, migrant depuis 1986. 30. Entretien n. 1975 à Pueblo (Colorado, EUA) au mois de mai 2005. Rodrigo, migrant depuis 1990.

\section{RÉSUMÉS}

Le présent article propose une exploration du rôle que jouent les aliments dans l'élaboration d'un discours identitaire dans un contexte de migration internationale. Dans le cadre de notre recherche doctorale, il s'est avéré que les récits des migrants autour de leur expérience de vie aux États-Unis faisaient souvent appel à la nourriture mexicaine pour manifester leur attachement à leur culture d'origine. Ce sentiment nostalgique à l'égard des aliments du pays d'origine est quelque peu paradoxal étant donné la présence notable d'aliments et de produits d'origine mexicaine dans les magasins et les restaurants des villes nord-américaines. Ce sentiment est d'autant plus paradoxal que ce qui circule le plus entre le village d'origine au Mexique et les différents points de résidence aux États-Unis sont des aliments. En même temps, intérioriser de nouvelles pratiques alimentaires est l'expression d'un signe de modernité par rapport aux habitudes culinaires du milieu rural mexicain d'où sont originaires ces migrants. Nous nous interrogerons dans ce sens sur la manière dont le migrant négocie son altérité à travers son alimentation. Nous verrons que c'est dans un rapport de proximité et/ou d'éloignement avec deux registres culinaires, que les sujets se servent de leur alimentation comme d'un support pour définir leur identité de migrant.

The present article proposes an exploration surrounding the role of food in the creation of an identity discourse. Through our doctoral research, we found that the stories of Mexican immigrants concerning their life experience in the United States often referenced food in order to express their attachment to their culture of origin. This nostalgic feeling for the food of their native culture is somewhat paradoxical because of the already important presence of Mexican foods and goods in stores and restaurants of North American cities. Food remains one of the most 
active product traffic networks between the Mexican village of origin and the different cities of residence in the United States. On the other hand, obtaining new dietary habits is often seen as a sign of modernity as opposed to the rural Mexican culinary customs of these migrants. In this perspective we analyze how migrants negotiate their otherness through the relationship they establish with both culinary customs, therefore using food as a way to define their own identity.

El presente artículo propone una exploración en torno al papel que tienen los alimentos en la elaboración de un discurso de tipo identitario en un contexto de migración internacional. En el marco de nuestra investigación de doctorado, observamos que al hablar de sus experiencias de vida en Estados Unidos, los migrantes hacían cotidianamente uso de la comida mexicana con el fin de manifestar el apego que mantienen con el lugar y su cultura de origen. Dicho sentimiento nostálgico es sin embargo un tanto paradoxal si consideramos la importante oferta de alimentos y productos de origen mexicano en tiendas y restaurantes de las ciudades norteamericanas. Sentimiento paradójico cuando sabemos que incluso las redes más activas de circulación entre el pueblo de origen y los diferentes puntos de destino migratorio en Estados Unidos son precisamente la de los alimentos. Al mismo tiempo, adquirir nuevos hábitos de alimentación parece constituir un elemento importante a partir del cual los migrantes definen una nueva manera de comer que es más moderna, la cual les permite distinguirse de los hábitos culinarios del terruño rural mexicano de donde son originarios. Nos interrogaremos en este sentido sobre la manera en que el migrante define su alteridad a través de su alimentación. Veremos que es según una relación de distancia o cercanía con respecto a dos diferentes registros culinarios que los sujetos utilizan la alimentación como un soporte para definir su identidad como migrantes.

\section{INDEX}

Mots-clés : pratiques alimentaires, changement alimentaire, migration, processus identitaires, réseaux sociaux, Mexique, États-Unis

Keywords : dietary habits, eating changes, immigration, identity process, social networks, Mexico, USA

Palabras claves : prácticas alimenticias, cambios alimentarios, migración, procesos identitarios, redes sociales, México, Estados Unidos

\section{AUTEUR}

\section{FRIDA CALDERON-BONY}

Frida Calderon-Bony est docteure en anthropologie de l'EHESS Paris et post-doctorante au Colegio de la Frontera Norte (COLEF) à Tijuana (Mexique). Email : fridacalatgmailpointcom. 